\title{
Proof and Computation in Geometry
}

\author{
Michael Beeson \\ San José State University, San José, CA
}

\begin{abstract}
We consider the relationships between algebra, geometry, computation, and proof. Computers have been used to verify geometrical facts by reducing them to algebraic computations. But this does not produce computer-checkable first-order proofs in geometry. We might try to produce such proofs directly, or we might try to develop a "backtranslation" from algebra to geometry, following Descartes but with computer in hand. This paper discusses the relations between the two approaches, the attempts that have been made, and the obstacles remaining. On the theoretical side we give a new first-order theory of "vector geometry", suitable for formalizing geometry and algebra and the relations between them. On the practical side we report on some experiments in automated deduction in these areas.
\end{abstract}

\section{Introduction}

The following diagram should commute:

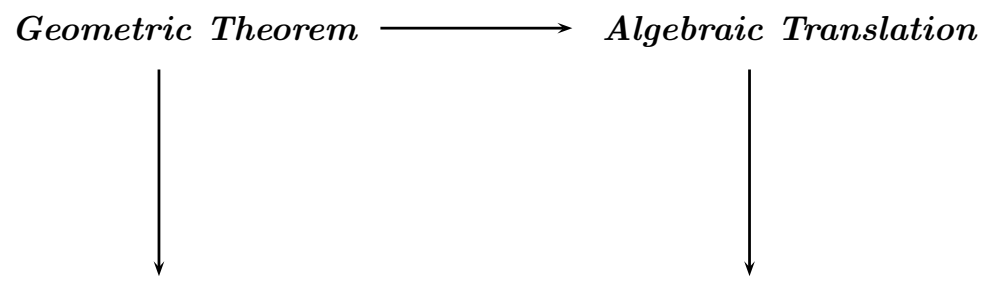

Geometric Proof

Algebraic Proof

That diagram corresponds to the title of this paper, in the sense that proof is on the left side, computation on the right. The computations are related to geometry by the two interpretations at the top and bottom of the diagram. In the past, much work has been expended on each of the four sides of the diagram, both in the era of computer programs and in the preceding centuries. Yet, we still do not have machine-found or even machine-checkable geometric proofs of the theorems in Euclid Book I, from a suitable set of first-order axioms-let alone the more complicated theorems that have been verified by computerized algebraic computations. ${ }^{1}$ In other words, we are doing better on the right side of the diagram than we are on the left.

\footnotetext{
${ }^{1}$ A very good piece of work towards formalizing Euclid is [1], but because it mixes computations (decision procedures) with first-order proofs, it does not furnish a counterexample to the statement in the text.
} 
First-order geometrical proofs are beautiful in their own right, and they give more information than algebraic computations, which only tell us that a result is true, but not why it is true (i.e. what axioms are needed and how it follows from those axioms). Moreover there are some geometrical theorems that cannot be treated algebraically at all (because their algebraic form involves inequalities).

We will discuss the possible approaches to getting first-order geometrical proofs, the obstacles to those approaches, and some recent efforts. In particular we discuss efforts to use a theorem-prover or proof-checker to facilitate a "back translation" from algebra to geometry (along the bottom of the diagram). This possibility has existed since Descartes defined multiplication and square root geometrically, but has yet to be exploited in the computer age. According to Chou et. al. ([8], pp. 59-60), "no single theorem has been proved in this way."

To accomplish that ultimate goal, we must first bootstrap down the left side of the diagram as far as the definitions of multiplication and square root, as that is needed to interpret the algebraic operations geometrically. We will discuss the progress of an attempt to do that, using the axiom system of Tarski and resolution theorem-proving.

\subsection{That commutative diagram, in practice}

In theory, there is no difference between theory and practice.

In practice, there is.

\section{- Yogi Berra}

Here is a version of the diagram, with the names of some pioneers ${ }^{2}$, and on the right the names of the computational techniques used in the algebraic computations arising from geometry. The dragon, as in maps of old, represents uncharted and possibly dangerous territory.

\section{Geometric Theorem $\longrightarrow$ Algebraic Translation}

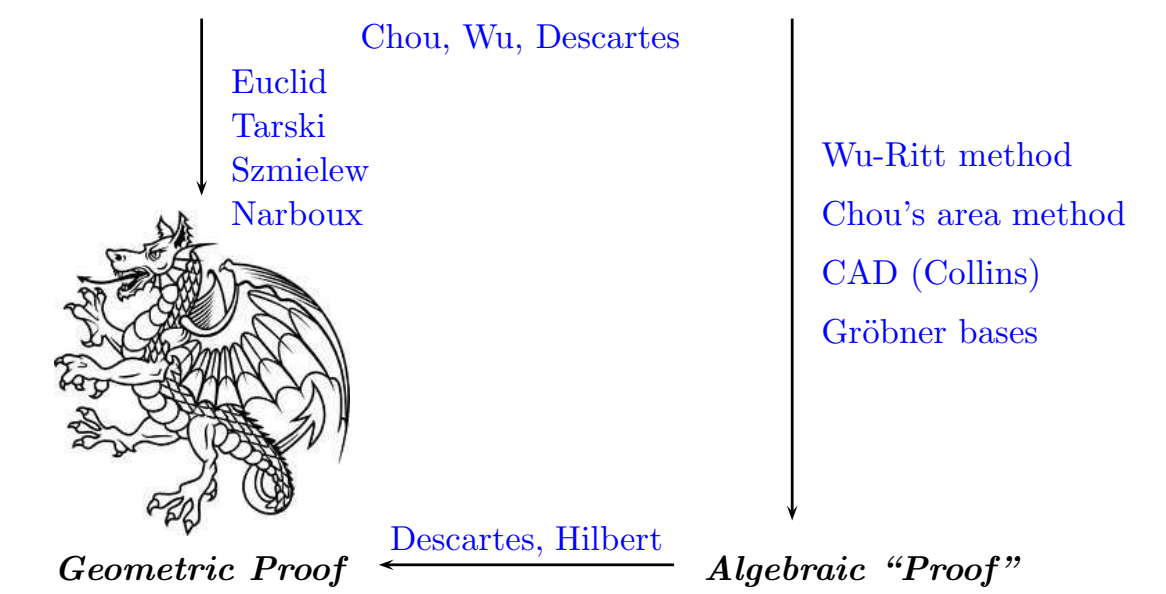

${ }^{2}$ Many others have contributed to this subject, including Gelernter, Gupta, Kapur, Ko, Kutzler and Stifter, and Schwabhäuser. 
Our proposal is the following:

(i) The goal is the lower left, i.e. first-order geometric proofs.

(ii) Finding them directly is difficult (hence the dragon in the picture).

(iii) Therefore: let's get around the dragons by going across the bottom from right to left.

\subsection{Issues raised by this approach}

The first issue is the selection of a language and axioms (that is, a theory) in which to represent geometrical theorems and find proofs. We discuss that briefly in the next section, and settle upon Tarski's language and the axioms for rulerand-compass geometry.

The next issue is this: if we want to go around the right side of the diagram and back across the bottom, and end up with a proof, then the computational part (the algebra on the right side of the diagram) will have to be formalized in some theory. In other words, we will have to convert computations to verified, or formal computations (proofs in some algebraic theory). ${ }^{3} \mathrm{~A}$ formal theory of algebra will be required.

The third issue is, how can we connect the left and right sides of the diagram? If we have a formal geometrical theory on the left, and a formal algebraic theory on the right, we need (at the least) two formal translation algorithms, one in each direction. Technically such mappings (taking formulas to formulas) are called "interpretations"; we will need them to take proofs to proofs as well as formulas to formulas.

That approach promises to be cumbersome: two different formal theories, two formal interpretations, algebraic computations, and proofs verifying the correctness of those computations. We will cut through some of these complications by exhibiting a new formal theory VG of "vector geometry." This theory suffices to formalize the entire commutative diagram, i.e. both algebra and geometry. The first half of this paper is devoted to the formal theories for geometry, algebra, and vector geometry, and some metatheorems about those theories.

Within that theoretical framework, there is room for a great deal of practical experimentation. We have carried out some preliminary experiments, on which we report. In these experiments, we used the resolution-based theorem provers Otter and Prover9, but that is an arbitrary choice; one could produce proofs by hand using Coq as in $[20]^{4}$ or in another proof-checker, or using another theorem-prover.

${ }^{3}$ This is related to the general problem of verifying algebraic computations carried out by computer algebra systems, which often introduce extra assumptions that occasionally result in incorrect results.

${ }^{4}$ There is an issue about how easy it is or is not to extract first-order geometric proofs from a Coq proof. In my opinion it should be possible, but Coq proofs are not prima facie first-order. 


\section{First order theories of geometry}

In this section we discuss the axiomatization of geometry, and its formalization in first-order logic. These are not quite the same thing, as there is a long history of second order axiomatizations (involving sets of points). Axiomatizations have been given by Veblen [33], Pieri [24], Hilbert [14], Tarski [31], Borsuk and Szmielew [5], and Szmielew [29], and that list is by no means comprehensive.

The following issues arise in the axiomatization of geometry:

- What are the primitive sorts of the theory?

- What are the primitive relations?

- What (if any) are the function symbols?

- What are the continuity axioms?

- How is congruence of angles defined?

- How is the SAS principle built into the axioms?

- How close are the axioms to Euclid?

- Are the axioms few and elegant, or numerous and powerful?

- Are the axioms strictly first-order?

- Can the axioms be stated in terms of the primitives, or do they involve defined concepts?

- Do the axioms have a simple logical form (e.g. universal or $\forall \exists$ )?

Evidently there is no space to discuss even the few axiomatizations mentioned above with respect to each of these issues; we point out that the answers to these questions are more or less independent, which gives us at least $n=2^{11}$ different ways to formalize geometry, whose relationships and mutual interpretations can be studied. Nearly every possible combination of answers to the "issues" has something to recommend it. For example, Hilbert has several sorts, and his axioms are not strictly first-order; Tarski has only one sort (points) and ten axioms. My own theory of constructive geometry $[4,3]$ has points, lines, and circles, and function symbols so that the axioms are quantifier-free and disjunction-free.

In Euclid, geometry involves lines, line segments, circles, arcs, rays, angles, and "figures" (polygons). Rays and segments are needed only for visual effect, so a formal theory can dispense with them.

Hilbert [14] treated angles as primitive objects and angle congruence as a primitive relation. But angles can be treated as ordered triples of points, so they too can be dispensed with, as we will now show. ${ }^{5}$ The key idea is Tarski's "five-segment axiom" (A5), shown in Fig. 1.

If the four solid segments in Fig. 1 are pairwise congruent, then the fifth (dotted) segments are congruent too. This is essentially SAS for triangles $d b c$ and $D B C$. The triangles $a b d$ and $A D E$ are surrogates, used to express the congruence of angles dbe and $D B E$. By using Axiom A5, we can avoid all mention of angles.

In fact, we don't even need lines and circles; every theorem comes down to constructing some points from given points, so that the constructed points

${ }^{5}$ The idea to define these notions (instead of take them as primitive) goes back (at least) to J. Mollerup [19], but he attributes it to Veronese. 
Fig. 1. The five-segment axiom (A5)
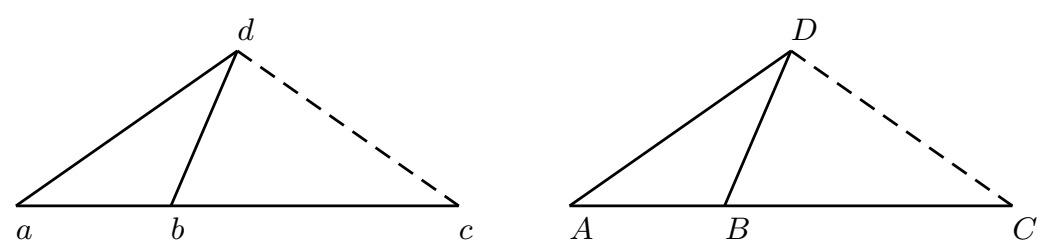

bear certain relations to the original points. Realizing this, Tarski formulated (in 1926) his theories of geometry using only one sort of variables, for points.

The fundamental relations to be mentioned in geometry are usually (at least for the past 120 years) taken to be betweenness and equidistance. We write $B(a, b, c)$ for " $a, b$, and $c$ are collinear, and $b$ is strictly between $a$ and $c$." Similarly $T(a, b, c)$ for non-strict betweenness: either $B(a, b, c)$ or $a=b$ or $b=c$. $T$ stands for "Tarski"; Hilbert used strict betweenness. ${ }^{6}$ Equidistance is formally written $E(a, b, c, d)$, but often written informally as $a b \equiv c d$ or $a b=c d$.

The question of function symbols is related to the issue of logical form. For example, we may wish to introduce $\operatorname{ext}(a, b, c, d)$ to stand for the point extending segment $a b$ past $b$ by the amount $c d$. In this way, we can reduce a $\forall \exists$ axiomatization to a universal one.

The question of continuity axioms brings us to the distinction between second order and first-order axiomatizations. Tarski seems to have been the first to give a first-order account of Euclidean geometry by restricting his continuity schema to first-order instances. His paper [31] is called What is Elementary Geometry, and he called his first-order theory "elementary geometry" to emphasize its first-order nature. We call this theory "Tarksi geometry". Because "elementary" means first-order, the word is not available for what is usually now known as "ruler and compass geometry." 7 In ruler and compass geometry, the infinite schema of all first-order continuity axioms is replaced by two consequences, "line-circle continuity" and "circle-circle continuity."

In the rest of this paper, we will work with Tarski's axioms for ruler and compass geometry. These axioms are known as A1 through A10, plus the linecircle and circle-circle continuity axioms. We chose to work with this theory because among geometrical theories, it is the simplest in the sense of having only one sort of variables, two primitive relations, and a small number of axioms that do not need defined notions to express. Starting with such pristine axioms requires a long development to reach the level of Euclid; this formal development was carried out by Tarski in his 1956-57 lecture notes, starting from a larger set

\footnotetext{
${ }^{6}$ Betweenness, which does not occur in Euclid, was introduced by Moritz Pasch [21]; see also [16] for another early paper on betweenness.

7 That would have otherwise been natural, since Euclid's work is titled the Elements. We can't call ruler and compass geometry "Euclidean", either, since that has come to mean geometry with the parallel axiom, as opposed to "non-Euclidean geometry."
} 
of axioms; and then by 1965 the axioms were reduced to A1-A10 plus continuity. For a history of this development see [32] or the foreword to [29].

\section{$3 \quad$ Fields and Geometries}

In this section we briefly review the known results connecting formal theories of geometry with corresponding formal theories of algebra (field theory).

A Euclidean field is an ordered field in which every positive element has a square root; or equivalently (without mentioning the ordering), a field in which every element is a square or minus a square, every element of the form $1+x^{2}$ is a square, and -1 is not a square.

If $\mathbb{F}$ is a Euclidean field, then using analytic geometry we can expand $\mathbb{F}^{2}$ to a model of ruler and compass geometry.

Descartes and Hilbert showed, by giving geometric definitions of addition, multiplication, and square root, that every model of Euclidean geometry is of the form $\mathbb{F}^{2}$, where $\mathbb{F}$ is a Euclidean field.

Fig. 2. Multiplication according to Hilbert

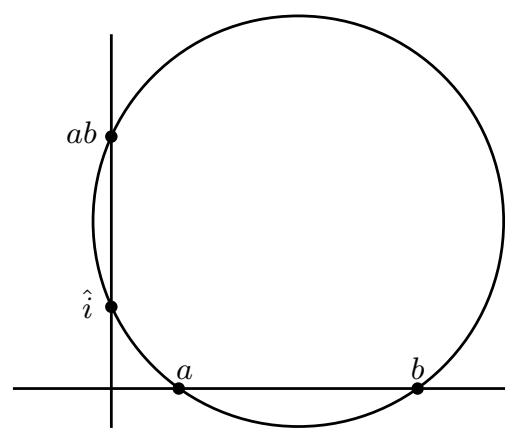

Similarly every model of Tarski geometry is $\mathbb{F}^{2}$, where $\mathbb{F}$ is real-closed. The smallest model of Tarski geometry corresponds to the case when $\mathbb{F}$ is the field of real algebraic numbers. The smallest model of ruler and compass geometry is the "Tarski field" $\mathbb{T}$, defined as the least subfield of the reals closed under square roots of positive elements. In a natural sense, $\mathbb{T}^{2}$ is the minimal model of ruler and compass geometry EG. $\mathbb{T}$ consists of all real algebraic numbers whose degree over $\mathbb{Q}$ is a power of 2 .

\subsection{Models and interpretations}

In general model-theoretic arguments are looked at by proof theorists as "interpretations." An interpretation maps formulas $\phi$ of the source theory into 
formulas $\hat{\phi}$ of the target theory, preserving provability:

$$
\vdash \phi \Rightarrow \vdash \hat{\phi}
$$

Usually the proof also shows how to transform the proofs efficiently. Generally interpretations have several advantages over models, all stemming from their greater explicitness. The main advantage is that an interpretation enables one to translate proofs from one theory to another. A model theoretic theorem, coupled with the completeness theorem, may imply the existence of a proof, but not give the slightest clue how to find it; while interpretations often give a linear-time proof translation.

There is a price to be paid, as the technical details of interpretations are often more intimidating than those of the corresponding model-theoretic arguments. Nevertheless, if we hope to use the equivalences of geometry and algebra to find proofs, model theory will not suffice. We need explicit interpretations.

Another reason for working with interpretations is that they can also be used for theories with non-classical logics, for example intuitionistic logic. The details of the interpretations between geometry and field theory can be found in [3]. Below, after introducing a theory of vector geometry, we will give a sample of these details.

\subsection{Interpretations between geometry and algebra}

Here we sketch the main ideas connecting formal theories of geometry and algebra, indicating how these ideas can be expressed using interpretations rather than model theory. Since at this point we have not given an explicit list of axioms, the discussion cannot be completely precise, but still the ideas can be explained. When we go from algebra to geometry, we fix a line $L$, which we call the " $x$-axis", containing two fixed distinct points $\alpha$ and $\beta$. (These interpret the scalars 0 and 1.) Then we show that one can construct a line perpendicular to any given line $K$, passing through a given point $q$, without needing a case distinction as to whether $q$ is or is not on $K$. Since we do not have variables for lines, we need two points (say $k_{1}$ and $k_{2}$ ) to specify $K$ and two to specify the resulting line, so we need two terms $\operatorname{perp}_{1}\left(k_{1}, k_{2}, q\right)$ and $\operatorname{perp}_{2}\left(k_{1}, k_{2}, q\right)$ that determine this perpendicular. Then we define "the $y$-axis" to be the perpendicular to the $x$-axis at $\alpha$. We can then define coordinate functions $X$ and $Y$ by terms of our geometrical theory, such that for any point $q, X(q)$ is a point $P$ on the fixed line $L$ such that the line containing $X(q)$ and $q$ is perpendicular to $L$, and the line containing $q$ perpendicular to the $y$-axis meets the $y$-axis at a point $Q$ such that $Q \alpha \equiv \alpha Y(q)$. (Notice that $Q$ is on the $y$-axis but $Y(q)$ is on the $x$-axis.) It is not at all trivial to construct these terms without a test-for-equality function (symbol), but it can be done (see [3]). ${ }^{8}$ Then we can find a term $F$ of our

\footnotetext{
8 This permits us to eschew a test-for-equality symbol, which is good, for two reasons: nothing like a test-for-equality construction occurs in Euclid, and simpler is better. But [3] uses terms for the intersections of lines and circles; whether those can be eliminated is not known.
} 
geometrical theory that takes two points $x$ and $y$ on the $x$-axis and constructs the point $p$ whose coordinates are $x$ and $y$. One first constructs the point $Q$ on the $y$-axis such that $\alpha Q \equiv \alpha y$, and the the lines perpendicular to the $x$ axis at $x$ and perpendicular to the $y$ axis at $Q$. One needs the parallel postulate (A10) to prove that these lines actually meet in the desired point $F(x, y)$.

Part of the price to pay for using interpretations instead of models is that the algebraic interpretation $\phi^{\star}$ of a geometric formula $\phi$ has two free variables for each free variable of $\phi$, one for each coordinate. Then when we translate back into geometry, these two variables do not recombine, but become two different point variables, restricted to the $x$-axis. They must be recombined using $F$.

\subsection{Euclid lies in the AE fragment}

By the AE fragment, or the $\forall \exists$ fragment, we mean the set of formulas of the form $\forall x \exists y A(x, y)$, where $x$ and $y$ may stand for zero or more variables, and $A$ is quantifier-free.

Euclid's theorems have the form,

Given some points bearing certain relations to each other, there exist (one can construct) certain other points bearing specified relations to the original points and to each other.

The case where no additional points are constructed is allowed. The points are to be constructed with ruler and compass, by constructing a series of auxiliary points. Constructed points are built up from the intersections of lines and circles.

Theorems of this form can be translated into Euclidean field theory (formulated with a function symbol for square root). Since the intersections of lines and circles, and the intersections of circles, can be expressed using only quadratic equations, and there is a function symbol for square root, constructed points correspond to terms of the theory. Euclid's theorems are thus in $\forall \exists$ form, both before and after translation into algebra.

A careful analysis of Euclid's proofs shows that, apart from some case distinctions as to whether two points are equal or not, or a point lies on a line or not, the proofs are constructive: Euclid provides a finite number of terms, one of which works in each case. This is closely related to Herbrand's theorem, which would tell us that if $\forall x \exists y A(x, y)$ is provable, then there are finitely many terms $t_{1}, \ldots, t_{n}$ such that the disjunction of the $A\left(x, t_{i}(x)\right)$ is provable.

Some parts of Euclid are about "figures", which are essentially arbitrary polygons. Euclid did not have the language to express these theorems precisely, since that would require variables for finite sets or lists or points, but we regard them as "theorem schemata", i.e. for each fixed number of vertices, we have a Euclidean theorem.

The AE fragment of the modern first-order theory of ruler and compass geometry is thus the closest thing we have to a formal analysis of Euclid. Euclid did not study theorems with more alternations of quantifiers. 


\subsection{Decidability issues}

A proper study of the relations between proof and computation in geometry must take place against the backdrop of the many known, and a few unknown, results about the decidability or undecidability of various theories. After all, the decidability of a formal theory means that provability in the theory can be reduced to computation. We offer in this section a summary of these known and unknown results.

Gödel and Church showed that in number theory, provability cannot be reduced to computation; Tarski showed that in geometry, it can, in the sense that Tarski geometry can be reduced to the theory RCF of real closed fields, for which Tarksi gave a decision procedure. Later Fischer and Rabin [10] showed that any decision procedure for RCF is at least exponential in the length of the input, and others showed it is at least double exponential in the number of variables; and Collins gave a decision procedure that is no worse than that bound (Tarski's was). (See [27] for more details.) Thus from a practical point of view it doesn't do us any good to know that RCF is decidable. There are interesting questions that can be formulated in RCF, questions whose answers we do not know, but if they involve more than six variables, then we are not going to compute the answers by a decision procedure for RCF.

On the other hand, if we drop the continuity axioms entirely, we get back the complications of number theory. Julia Robinson [28] proved that $\mathbb{Q}$ is an undecidable field, and later extended this result to algebraic number fields. Regarding the decidability of theories rather than particular fields, Ziegler [36] proved that any finitely axiomatizable extension of field theory is undecidable-in particular the theory of Euclidean fields. His proof shows the AEA fragment is undecidable. (Here AEA means $\forall \exists \forall$, formulas with three blocks of unlike quantifiers as indicated.) It does not say anything about the $\mathrm{AE}$ fragment. It is presently an open problem whether the $\mathrm{AE}$ fragment of RCF (and hence the $\mathrm{AE}$ fragment of Tarski geometry) is decidable. The fact that Euclid's Elements lies within this fragment focuses attention on the problem of its decidability.

Tarski conjectured that $\mathbb{T}$ (recall that $\mathbb{T}^{2}$ is the smallest model of rule and compass geometry) is undecidable, but this is still an open problem. Since $\mathbb{T}$ is not of finite degree over the rationals, its undecidability is not implied by Julia Robinson's results about algebraic number fields.

\section{Tarski's ruler and compass geometry}

In this section we comment on the axioms of Tarski's theory, which can be found in full formal detail in [32] or [29]. This section is intended both as an introduction to Tarski's axioms, and as a description of the Skolem symbols we added to make the theory quantifier-free instead of $\forall \exists$. As mentioned above, the primitives are non-strict betweenness $T$ and segment congruence $a b \equiv c d$, which is a 4 -ary relation between points. 


\subsection{Tarski's first six axioms}

Axiom A5 has been discussed and illustrated above. The other five are

$$
\begin{array}{r}
u v \equiv v u \\
u v \equiv w x \wedge u v \equiv y z \rightarrow w x \equiv y z \\
u v \equiv w w \rightarrow u=v \\
T(u, v, \operatorname{ext}(u, v, w, x)) \\
T(u, v, u) \rightarrow u=v
\end{array}
$$

(A4), segment extension

We have added a Skolem symbol to express (A4) without a quantifier.

\subsection{Pasch's axiom (1882)}

Moritz Pasch [21] (See also [22], with an historical appendix by Max Dehn) supplied an axiom that repaired many of the defects that nineteenth-century rigor found in Euclid. Roughly, a line that enters a triangle must exit that triangle. As Pasch formulated it, it is not in AE form. There are two AE versions, illustrated in Fig. 4.2. These formulations of Pasch's axiom go back to Veblen [33], who proved outer Pasch implies inner Pasch. Tarski took outer Pasch as an axiom in [31].

Fig. 3. Inner Pasch (left) and Outer Pasch (right). Line $p b$ meets triangle $a c q$ in one side. The open circles show the points asserted to exist on the other side.
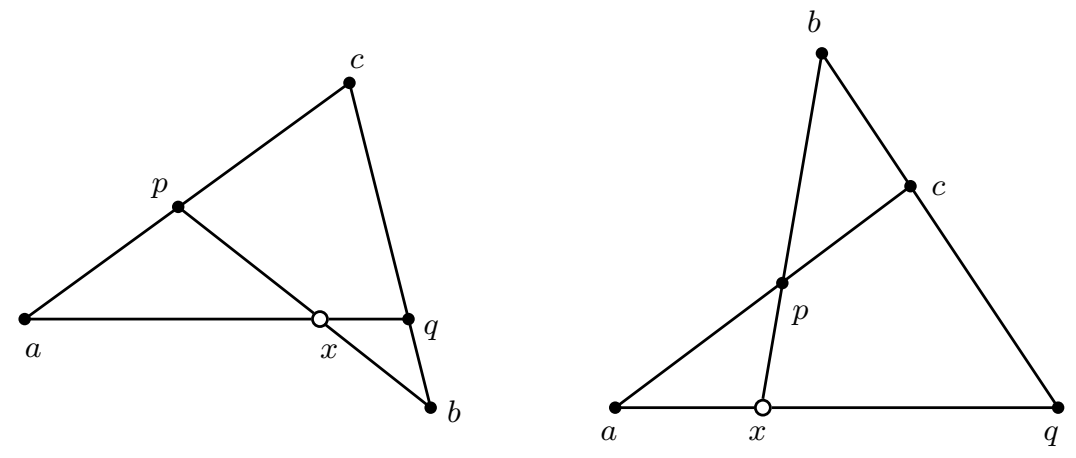

Tarski originally took outer Pasch as an axiom, but following the "final" version of Tarski's theory in [29], we take inner Pasch. Seeking a quantifier-free formulation, we introduce a function symbol $i p$ to produce the intersection points from the five points labeled in the diagram. When the betweenness relations in the diagram are not satisfied, nothing is asserted about the value of ip on those points. 


\subsection{Gupta's thesis}

In his 1965 thesis [12] under Tarski, H. N. Gupta proved two great theorems: ${ }^{9}$

(i) Inner Pasch implies outer Pasch. After that Szmielew's development used inner Pasch as an axiom (A7) and dropped outer Pasch (although Tarski was still arguing decades later [32] for the other choice).

(ii) Connectivity of Betweenness:

$$
a \neq b \wedge T(a, b, c) \wedge T(a, b, d) \rightarrow T(a, c, d) \vee T(a, d, c) .
$$

That is, betweenness determines a linear order of points on a line. Points $d$ and $c$, both to the right of $b$ on Line $(a, b)$, must be comparable.

The connectivity of betweenness was taken as an axiom by Tarski, but once Gupta proved it dependent, it could be dropped. Gupta never published his thesis, but his proof of connectivity appears as Satz 5.1 in [29]. The proof is complicated: it uses 8 auxiliary points and more than 70 inferences, and uses all the axioms A1-A7. His proof of outer Pasch (from inner Pasch) also occurs in [29] as Satz 9.6.

\subsection{Dimension Axioms}

(A8) (lower dimension axiom) says there are three non collinear points (none of them is between the other two)

(A9) (upper dimension axiom) says that any three points equidistant from two distinct points must be collinear. In other words, the locus of points equidistant from $a$ and $b$ is a line (not a plane as it would be in $\mathbb{R}^{3}$ ).

(A1) through (A9) are the axioms for "Hilbert planes."

\subsection{Tarski's Parallel Axiom (A10)}

In the diagram (Fig. 4), open circles indicate points asserted to exist. There are other equivalent forms; see $[32,29] .{ }^{10}$ We would need to introduce new function symbols to work with A10 in a theorem-prover. Since none of the work in this paper depends on the exact formulation of the parallel axiom, we do not discuss alternate formulations.

\subsection{Line-circle continuity}

In Fig. 5 , point $p$ is "inside" the circle since $a p \equiv a x$. Then the points indicated by open circles must exist.

${ }^{9}$ Gupta got his Ph. D. sixteen years after earning his second master's degree in India. There was at least one more great theorem in his thesis-I do not mean to imply that he proved only two great theorems.

${ }^{10}$ Tarski and Givant [32] label a certain quantifier-free formula "Third Form of Euclid's Axiom", which is misleading, because this formula is not equivalent to A10 (see Exercise 18.4 in [13]). 
Fig. 4. Tarski's parallel axiom

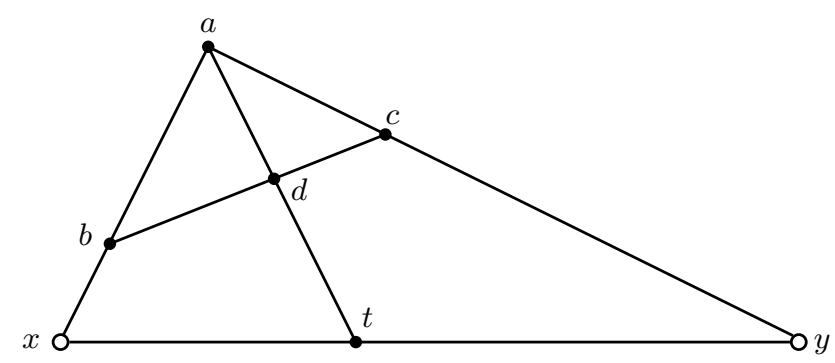

Fig. 5. Line-circle continuity. Line $L$ is given by two points $A, B$ (not shown). The points shown by open circles are asserted to exist.

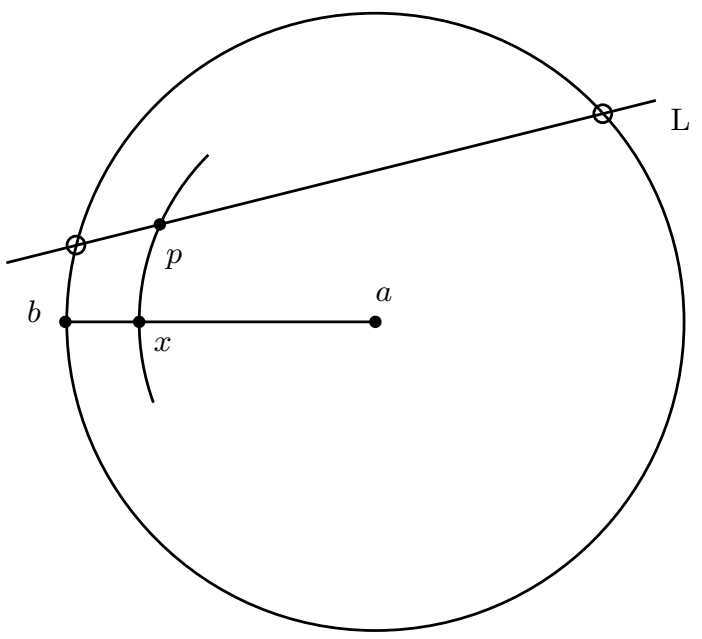

We use two function symbols $\ell c_{1}$ and $\ell c_{2}$ to name the points $\ell c_{1}(A, B, a, x, b, p)$ and $\ell c_{2}(A, B, a, x, b, p)$, where $A$ and $B$ are two unequal points that determine the line $L$. In [3], we also introduce another axiom, the point of which is to ensure that the two intersection points occur in the same order on $L$ as $A$ and $B$. This can be expressed as a disjunction of several betweenness statements, essentially listing the possible allowed orders of the four points. Since non-strict betweenness is used in this axiom, the points $p$ and $x$ might be on the circle, in which case the line is tangent to the circle and the two intersection points coincide. The additional axiom implies that the two intersection points each depend continuously on their arguments. Tarski used an existential quantifier instead of function symbols to formulate line-circle continuity, so the extra axiom was not needed; but we want a quantifier-free axiomatization, and the extra axiom is natural so that there will be one natural model $\mathbb{F}^{2}$ over each Euclidean field $\mathbb{F}$, instead of uncountably many with strange discontinuous interpretations of $\ell c_{1}$ and $\ell c_{2}$. 


\subsection{Circle-circle continuity}

In Fig. 6, points $p$ and $q$ on circle $K$ are "inside" and "outside" circle $C$, respectively, because $a x \equiv a p$ and $a x \equiv a q$. Then points 1 and 2 (indicated by open circles in the figure) exist, i.e. lie on both circles. The two intersection points can coincide in some degenerate cases, but if the two circles coincide, so there are more than two intersection points, then points 1 and 2 become "undefined", or technically, since we are not using the logic of partial terms, they just become unknown points about which we say nothing.

As for line-circle continuity, we introduce two Skolem functions $c c_{1}$ and $c c_{2}$ to define the intersection points. Since there are only point variables, circle $C$ in the figure will be given by its center $a$ and the point $y$, and circle $K$ is given by its center $b$ and the point $q$. Hence the arguments of the two Skolem functions are just the points labeled with letters in Fig. 6.

Fig. 6. Circle-circle continuity. $p$ is inside $C$ and $q$ is outside $C$, as witnessed by $x, y$, and $z$, so the intersection points 1 and 2 exist.

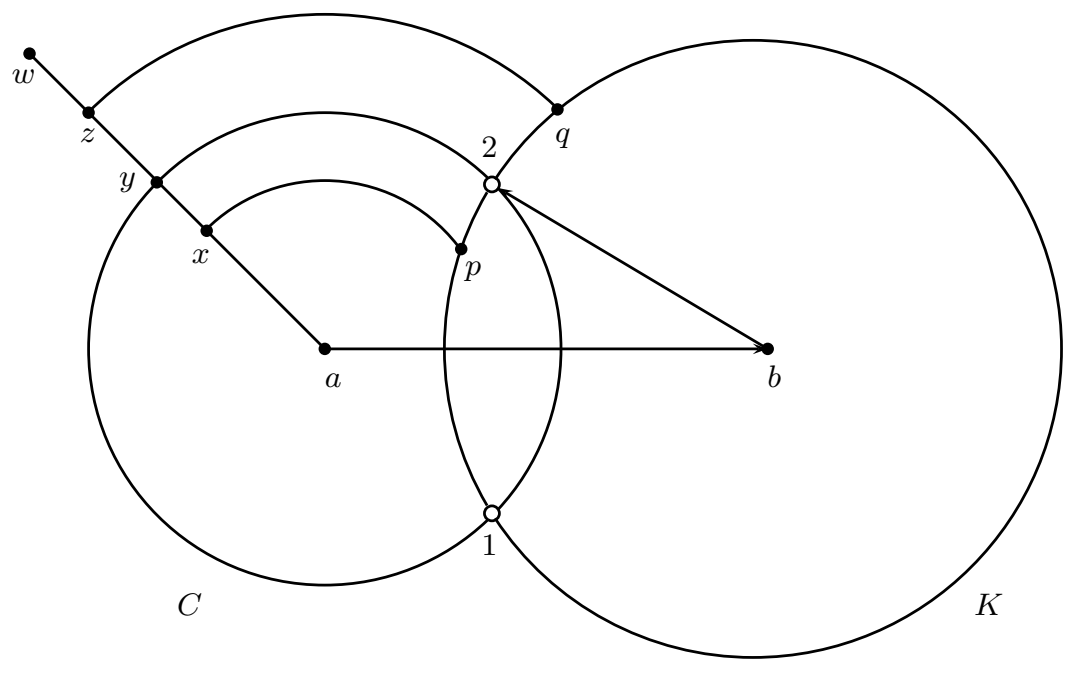

In general, when one introduces a Skolem function, one may lose completeness (perhaps that is why Tarski left his axioms in $\forall \exists$ form). Once we Skolemize the circle-circle continuity axiom, we also want extra axioms to distinguish the two points of intersection and ensure that they depend continuously on their arguments. The rule we want to state is that the "turn" from $a$ to $b$ to intersection point 1 is a right-hand turn, and the turn from $a$ to $b$ to intersection point 2 is a left-hand turn. Rather than defining "right turn" directly, we define $a b c$ to be a right turn if, when we draw the circles of radius $a c$ and center $a$, and radius $b c$ and center $b$, their first intersection point is $c$; if $c$ is instead the second intersection point, then $a b c$ is a left turn (by definition). Then we add an axiom 
saying that if $c$ and $d$ are on the same side of the line through $a$ and $b$, and $a b c$ is a right turn, so is $a b d$, and the same for left turns. For the definition of "same side", we follow [29], Definition 9.7, p. 71. Since these axioms play no role in this paper, we refer the interested reader to [3] for further details.

\section{VG, a formal theory of vector geometry}

In this section, we describe a first-order theory VG that contains both geometry and algebra. This theory permits us to formalize the relationships between algebra and geometry in both directions, and to formalize the Chou area method directly. The theories of algebra and of geometry become fragments of VG.

\subsection{Language of Vector Geometry}

Three sorts:

- points $p, q, a, b$

- scalars $\alpha, \beta, \lambda, s, t$

- vectors $\mathbf{u}, \mathbf{v}$

Intuitively you may think of vectors as equivalence classes of directed line segments under the equivalence relation of parallel transport. Constructors and accessors:

$-p \circ q$ is a vector, the equivalence class of directed segment $p q$.

- scalar multiplication: $\lambda \mathbf{u}$ is a vector

- dot product: $\mathbf{u} \cdot \mathbf{v}$ is a scalar

- cross product: $\mathbf{u} \times \mathbf{v}$ is a scalar (not a vector, we are in two dimensions)

\subsection{Language of Vector Geometry}

Relations:

- betweenness and equidistance from Tarski's language

- Equality for points, equality for vectors, equality for scalars. Technically these are different symbols.

$-x<y$ for scalars.

Function symbols (other than constructors and accessors) and constants:

- Skolem symbols for Tarski's language, specifically ext for segment extension, ip for inner Pasch, and Skolem symbols for line-circle and circle-circle continuity, as described above.

$-0,1, *,+, /$, unary and binary - , and $\sqrt{ }$ for scalars.

- $\mathbf{0}$ is a vector; $\mathbf{u}+\mathbf{v}, \mathbf{u}-\mathbf{v}$, and $-\mathbf{u}$ are vectors.

- $\hat{0}, \hat{1}$, and $\hat{i}$ are unequal points.

$-\hat{i}$ is a point equidistant from 1 and from $-1=\operatorname{ext}(1,0,0,1)$. 


\subsection{Division by zero and square roots of negative numbers}

$1 / 0$ is "some scalar" rather than "undefined", because we want to use theorem provers with this language and they do not use the logic of partial terms. One cannot prove anything about $1 / 0$ so it does not matter that it has some undetermined value. For example, we have the axiom $x \neq 0 \rightarrow x *(1 / x)=1$, not the axiom $x *(1 / x)=1$. Other "undefined" terms are treated the same way.

\subsection{Axioms of Vector Geometry VG}

- Tarski's axioms for ruler-and-compass geometry.

- The scalars form a Euclidean field.

- The obvious axioms for unary - and / and $\sqrt{ }$ permit us to avoid existential quantifiers in the axioms for Euclidean fields. The use of binary - is a convenience; the axiom is $a-b=a+(-b)$.

- The vectors form a vector space over the scalars.

- The usual laws for dot product and $2 \mathrm{~d}$ cross product.

$-a \circ b=-b \circ a$

$-p \circ p=\mathbf{0}$

- $E(\hat{0}, \hat{i}, \hat{0}, \hat{1})$

$-E(\hat{i}, \hat{1}, \hat{i},-\hat{1})$ where $-\hat{1}=\operatorname{ext}(\hat{1}, \hat{0}, \hat{1}, \hat{0})$

- If $a b$ and $c d$ are parallel and congruent, then $a \circ b= \pm c \circ d$, with the sign positive if segment $a d$ meets segment $b c$, and negative if it does not.

- If $a, b, c$, and $d$ are collinear and $a b$ and $c d$ are congruent, then $a \circ b= \pm c \circ d$, with the appropriate sign (given by betweenness conditions expressing the intuitive idea that the sign is positive if the directed segments $a b$ and $c d$ have the same direction).

The geometrical part (based on Tarski's axioms A1-A10 and line-circle and circle-circle continuity, but in a quantifier-free form) we call "Euclidean geometry" EG. There are function symbols for the intersection points of two lines, of a line and a circle, and of two circles; that is a slightly different choice of function symbols from the quantifier-free version of Tarski's axioms used in this paper. See [3] for a detailed formulation.

\subsection{Analytic geometry in VG}

This corresponds to the translations across the top and bottom of our (supposedly) commutative diagram. Let $\phi$ be a formula of EG. Let $\phi^{\star}$ be a translation of $\phi$ into Euclidean field theory (expressed using scalar variables in VG). In fact, more generally we can define $\phi^{\star}$ when $\phi$ is a formula of VG, not just of $\mathbf{E G}$.

The first thing to notice here is that there is more than one way to define such a translation $\phi$. The obvious one is the one that is taught to middle-school children, which we call the "Cartesian translation." Lines are given by linear equations, and points by pairs of numbers. In practice this leads to many case distinctions as vertical lines require special treatment. Another translation, invented by Chou [8], takes a detour through vectors, but can be expressed directly 
in VG, and then (whichever way we interpreted points), of course vectors can be expressed by coordinates using the scalars of VG. We will discuss the Chou translation in more detail below. The important fact, expressing the adequacy of VG for this part of the method, is the following theorem.

Theorem 1 (Analytic Geometry). Let $\phi$ be a formula of VG. Let $\phi^{\star}$ be either the Cartesian translation of $\phi$, or the Chou translation. Then

$$
\mathbf{V G} \vdash \phi \quad \text { implies } \quad \mathbf{E F} \vdash \phi^{\star}
$$

where $\mathbf{E F}$ is the theory of Euclidean fields.

Remark. This corresponds to the top of the commutative diagram.

Proof. Here we discuss only the Cartesian translation. By "the $x$-axis" we mean the line containing $\alpha$ and $\beta$, where $\alpha$ and $\beta$ are the two distinct points mentioned in the dimension axioms. Next we define the Cartesian interpretation $\phi^{\star}$ of $\phi$. To define $\phi^{\star}$, we have to first assign a term $t^{\star}$, or a pair of terms $\left(t_{1}, t_{2}\right)$, of $\mathbf{E F}$ for each term $t$ of EG. In fact, we define $t^{\star}$ for all terms of VG, not just EG. Since points and vectors are to be interpreted as pairs of scalars, we use pairs for terms of those types; for a term of $\mathbf{E F}$ we just have $t^{\star}=t$. Otherwise we write $t^{\star}=\left(t_{1}^{\star}, t_{2}^{\star}\right)$. When $t$ is a point variable $x$, then $x$ occurs in the official list of all point variables as the $n$-th entry, for some $n$, and we define $x^{\star}$ to be the pair consisting of the scalar variables occurring with indices $4 n$ and $4 n+1$ in the official list of scalar variables. Similarly for vector variables, but using indices $4 n+2$ and $4 n+3$.

The definitions of $\phi^{\star}$ for the case of atomic formulas involving betweenness or segment congruence is straightforward analytic geometry. For details see [13] or [3]. To extend the definition of $\phi^{\star}$ from EG to VG, we have to show that dot product and cross product of vectors can be algebraically defined. For example,

$$
(t \times s)^{\star}=\left(t_{1}^{\star} s_{2}^{\star}-t_{2}^{\star} s_{1}^{\star}\right)
$$

Once $t^{\star}$ is defined, then $\phi^{\star}$ commutes with the logical connectives and quantifiers, except that for quantifiers, each point or vector variable is "doubled", i.e. changed to two scalar variables.

Now the theorem is proved by induction on the length of proofs in VG. The base case is when $\phi$ is an axiom of VG. We verify in EF that the algebraically defined relations of betweenness and equidistance satisfy the axioms of EG. That is lengthy, and not entirely straightforward, in the case of circle-circle continuity (but note that the same complications occur whether we are using model theory or interpretations). See, for example, [13], page 144. See also [3] for some details omitted in [13]. We note that if $\phi$ is quantifier-free (or AE) in EG, then $\phi^{\star}$ is also quantifier-free (or AE).

\subsection{Geometric arithmetic in VG}

Along the bottom of the commutative diagram, we have a translation $\phi^{\circ}$ from EF to EG, following Descartes with improvements by Hilbert. We show that 
this translation can be extended to be defined on terms and formulas of VG, not only of EF. We fix a particular line $L$ (given by the two unequal points $\alpha$ and $\beta$, which we use as the interpretations of 0 and 1 , respectively). Scalars of VG are interpreted as points on $L$, i.e. collinear with $\alpha$ and $\beta$. Vectors are interpreted as pairs of such points. Since there is no pairing function in $\mathbf{E G}$, the formula $\phi^{\circ}$ may have more variables than $\phi$, as each vector variable converts to two point variables. The terms $(t+s)^{\circ},(t-s)^{\circ},(-t)^{\circ},(t \cdot s)^{\circ}$, and $(\sqrt{t})^{\circ}$, are defined using terms of EG. For example, the definition of $(t \cdot s)^{\circ}$ should be the one suggested by Fig. 2 .

It is not at all obvious that $t^{\circ}$ can be defined using the terms of $\mathbf{E G}$, which do not include a symbol for definition-by-cases; in other words there are no terms in EF to construct a term $d(a, b)$ that is equal to $\alpha$ if $a=b$ and to $\beta$ otherwise. But the definitions of addition and multiplication given by Descartes require such a case distinction; Hilbert's multiplication does not, but his addition still does. We have shown in [3] that an improved (continuous) definition of addition can be given; there only constructive logic is used, but here that is not an issue. If that were not true, we would simply include a definition-by-cases symbol in EG, and our main results would not be affected; but it is not necessary. For complete details of the interpretation from EF to EG, see [3].

Theorem 2 (Geometric algebra). Let $\phi$ be a formula of EG. Let $\phi^{\circ}$ be the translation discussed above of VG into EG. Then

$$
\begin{array}{rll}
\mathbf{V G} \vdash \phi & \text { implies } & \text { EG } \vdash \phi^{\circ} \\
\mathbf{V G} \vdash \phi & \text { implies } & \text { EG } \vdash\left(\phi^{\star}\right)^{\circ}
\end{array}
$$

Remark. This corresponds to the bottom of the commutative diagram.

Proof sketch. It has to be verified geometrically that multiplication, addition, and square root satisfy the laws of Euclidean field theory. This goes back to Descartes and Hilbert, but as noted above, since we do not have a test-for-equality function in EG, a more careful definition of addition is required. Since we have extended the interpretation to $\mathbf{V G}$, we also need to verify the laws of vector spaces and of cross product and dot product geometrically. It is possible to do this directly, but we can also circumvent the need for those details, by defining $\phi^{\circ}$ to be $\left(\phi^{\star}\right)^{\circ}$ for formulas $\phi$ involving vectors. Note that the language of VG has no terms constructing vectors from points, so if $\phi$ contains terms of type vector, it does not contain betweenness or equidistance or any subterms of type point. Hence it is not actually necessary to directly verify the laws of cross product and dot product and vector spaces geometrically.

\subsection{Conservativity and commutativity}

Suppose we start with a geometric theorem $\phi$ and somehow prove either it or $\phi^{\star}$ with the aid of analytic geometry. (By Theorem 1, if we have a proof of $\phi^{\star}$, we can get a proof of $\phi$, and vice-versa.) Then can we eliminate the "scaffolding" of analytic geometry, and find a purely geometric proof of $\phi$ ? Yes, we can: 
Theorem 3. VG is a conservative extension of $\mathbf{E G}$. That is, if $\phi$ is a formula in the language of Tarski's geometry $\mathbf{E G}$, and $\mathbf{V G}$ proves $\phi$, then $\mathbf{E G}$ proves $\phi$.

Proof. Suppose $\phi$ is a formula of EG, and VG proves $\phi$. Then by Theorem 2, $\phi^{\circ}$ is provable in EG. But $\phi^{\circ}$ is $\phi$ since $\phi$ is a formula of EG. That completes the proof of the theorem.

Suppose we start with a formula $\phi$ of Euclidean field theory, and find a proof of it using vectors, or even using the full apparatus of VG. Then it is already provable from the axioms of Euclidean fields:

Theorem 4. VG is a conservative extension of Euclidean field theory $\mathbf{E F}$.

Proof. Suppose $\phi$ is a formula of Euclidean field theory EF, and suppose VG proves $\phi$. Then $\phi^{\star}$ is provable in EF. But by definition of $\phi^{\star}$, when $\phi$ is a formula of $\mathbf{E F}, \phi^{\star}$ is exactly $\phi$. Hence $\mathbf{E F}$ proves $\phi$. That completes the proof.

Next we show that the two interpretations $\phi^{\star}$ and $\psi^{\circ}$ are, up to provable equivalences, inverses. This is by no means immediate, since the definitions have no apparent relation to one another. But nevertheless, they both express the same geometric relationships.

Theorem 5 (Commutativity). Let $\phi$ be a formula of EG with no free variables. Then

$$
\left(\phi^{\star}\right)^{\circ} \leftrightarrow \phi
$$

is provable in EG. Similarly, if $\psi$ is a theorem of $\mathbf{E F}$ with no free variables, then

$$
\left(\psi^{\circ}\right)^{\star} \leftrightarrow \psi
$$

is provable in $\mathbf{E F}$.

We will not give a proof of this theorem here, as it is highly technical. The theorem has to be first formulated in a way that holds for formulas with free variables, as well as for formulas without, and then proved by induction on the complexity of $\phi$. The technical issue here is the "doubling" of variables when we pass from point variables to scalar variables, and the "uncoordinatizing" in the other direction. We will explain what "uncoordinatizing" means next.

We will illustrate the proof by explaining one example, the case when $\phi$ is $T(\alpha, y, \beta)$. Then the point variable $y$ is "doubled" by $\phi^{\star}$ to the two scalar variables $\left(\lambda_{1}, \lambda_{2}\right)$, intuitively representing the coordinates of $y$. (Defining this precisely depends on setting up a one-to-two correspondence between the lists of variables of type point and type scalar.) Then $\phi^{\star}$ is

$$
\lambda_{2}=0 \wedge 0 \leq \lambda_{1} \wedge \lambda_{1} \leq 1 .
$$

Now going back to geometry, the two scalar variables do not convert back to one point variable. Instead they become two variables of type point, $y_{1}$ and $y_{2}$, restricted (by $\left.\left(\phi^{\star}\right)^{\circ}\right)$ to lie on the $x$-axis (the line through $\alpha$ and $\beta$ ). We write $\operatorname{Col}(x, y, z)$ for " $x, y$, and $z$ lie on the line containing distinct points $x$ and $y$ ", 
defined in terms of betweenness. Then $\left(\phi^{\star}\right)^{\circ}$ is equivalent to (though not literally the same as)

$$
\operatorname{Col}\left(\alpha, \beta, y_{1}\right) \wedge \operatorname{Col}\left(\alpha, \beta, y_{2}\right) \wedge y_{2}=\alpha \wedge T\left(\alpha, y_{1}, \beta\right)
$$

Finally we construct the point $q=F\left(y_{1}, y_{2}\right)$ using the $F$ described above. Then $X(q)=y_{1}$ and $Y(q)=y_{2}$. Then

$$
\begin{aligned}
\left(\phi(y)^{\star}\right)^{\circ} & \leftrightarrow\left(\left(\phi^{\star}\right)\left(\lambda_{1}, \lambda_{2}\right)\right)^{\circ} \\
& \leftrightarrow \phi(q) \\
\left(\phi(y)^{\star}\right)^{\circ} & \leftrightarrow \phi\left(F\left(y_{1}, y_{2}\right)\right)
\end{aligned}
$$

Here $y_{1}$ and $y_{2}$ are point variables related to the original point variable $y$ by this rule: if $y$ is the $n$-th point variable $x_{n}$, then $y_{1}$ is $x_{2 n}$ and $y_{2}$ is $x_{2 n+1}$. Now the variables on the left side of (1) are not related to the variables on the right in any semantic way; to state the commutativity theorem for $\phi$ we need this:

$$
y=F\left(y_{1}, y_{2}\right) \rightarrow\left(\left(\phi(y)^{\star}\right)^{\circ} \leftrightarrow \phi(y)\right)
$$

where in spite of appearances the formula $\left(\left(\phi(y)^{\star}\right)^{\circ}\right.$ contains $y_{1}$ and $y_{2}$ free, not $y$. Equation (2) demonstrates the way that $\phi \leftrightarrow\left(\phi^{\star}\right)^{\circ}$ is generalized to formulas with free variables. Granted, this is technical, but it works and is in some sense natural, and it is the price we have to pay for the benefits of an explicit interpretation. Once this is correctly formulated, the rest of the proof is straightforward (which is not the same thing as "short").

\subsection{Algebra in VG}

When we "prove" a geometric theorem by making a corresponding algebraic computation, we have not yet proved anything; we have only made a computation. In order to convert such a computation (ultimately) to a geometric proof, it will first be necessary to convert an algebraic computation to an algebraic proof. This also goes under the name of "verifying a computation", or sometimes, "verifying the correctness of a computation."

What we want to prove is that "computationally equal" terms $t$ and $s$ are provably equal. For example, we can compute by simple algebra that

$$
\left(x^{2}-y^{2}\right)^{2}+4 x^{2} y^{2}=\left(x^{2}+y^{2}\right)^{2}
$$

But that does not deliver into our hands a formal proof of that equation from the axioms of Euclidean field theory.

The principal problem here is that "computationally equal" is not very well defined. One is at first tempted to say: if your favorite computer algebra system says the terms are equal, they are computationally equivalent. But if we take that definition, then it is false that computationally equivalent terms are provably equal. For example, Sage and Mathematica agree that $x \cdot(1 / x)=1$, but that is 
not provable in $\mathbf{E F}$, since, if it were, we would have $0 \cdot 1 / 0=1$, but since $0 \cdot z=0$ we also have $0 \cdot 1 / 0=0$, hence $1=0$, but $1 \neq 0$ is an axiom of $\mathbf{E F}$.

Such problems arise from the axioms of $\mathbf{E F}$ that are not equational, for example $x \neq 0 \rightarrow x \cdot(1 / x)=1$ and $x \geq 0 \rightarrow(\sqrt{x})^{2}=x$. If we use these equations without regard to the preconditions, false results can be obtained.

We do not know how to define "computationally equivalent terms" except by provability of $t=s$ in $\mathbf{E F}$, or more generally, the vector and scalar part of VG. The search for a theorem or general result degenerates to a practical problem: given a computation by a computer algebra system that $t=s$, determine the minimal "side conditions" $\phi$ on the variables of $t=s$ necessary for the provability of $t=s$ and find a first-order proof of $t=s$. One may or may not wish to consider paramodulation steps as legal.

\subsection{Chou's method formalizable in VG}

This subsection presumes familiarity with Chou's method [8]. Readers without that prerequisite may skip this subsection and continue reading, but since Chou's method is an important method of proving geometrical theorems by algebraic computations, we want to verify that it can be directly formalized in VG.

We start with Chou's basic concept, the position ratio. In VG, we define

$$
\frac{a b}{c d}:=\operatorname{pr}(a, b, c, d)=\frac{(a \circ b) \cdot(c \circ d)}{(c \circ d) \cdot(c \circ d)}
$$

Our $p r$ is defined whenever $c \neq d$. Chou's position ratio is defined only when $a$, $b, c$, and $d$ are collinear, but in that case they agree.

Chou makes extensive use of the signed area of an oriented triangle. We define that concept in VG by

$$
\mathcal{A}(p, q, r):=\frac{1}{2}(q \circ p) \times(q \circ r) .
$$

Chou's other important concepts and theorems can also be defined and proved in VG. In particular, the co-side theorem can be proved in VG. This should be checked by machine; it would make a good master's thesis.

\section{Finding formal proofs from Tarski's Axioms}

An essential part of the project of making our diagram commute is to find formal proofs in geometry up to the point where multiplication, addition, and square root can be geometrically defined and their field-theoretic properties proved. We chose to attack this project using Tarski's axioms and resolution theorem provers. One of the reasons for this choice is the existence of a very detailed "semiformal" development due to Szmielew. ${ }^{11}$ Another reason is that others have tried in the

\footnotetext{
${ }^{11}$ Wanda Szmielew developed course notes for her course in the Foundations of Geometry at UC Berkeley, 1965-66, and gave a copy to her successor as instructor of that course, Wolfram Schwäbhauser. These notes incorporated important contributions from Gupta's thesis [12], including the two theorems mentioned above. After her death, her notes were published with "inessential changes" as part of [29].
} 
past to work with resolution theorem provers and Tarski's axioms. Specifically, MacPharen, Overbeek, and Wos [18] worked 37 years ago with Tarski's 1959 system; after the publication of [29], Quaife $[25,26]$ used Otter to formalize the first four of the fifteen chapters of Szmielew's development (that is, Chapters 2-5 out of 2-16). Quaife solved some, but not all, of the challenge problems from [18], and added some challenge problems of his own. In 2006, Narboux [20] checked Szmielew's proofs using Coq, up through Chapter 12. While this is fine work, the fact remains that after almost forty years, we have collectively still not produced computer-checked proofs of Szmielew's development-whether by an automated reasoning program (such as Otter), an interactive proof-checker (such as Coq), or by any other means. ${ }^{12}$

Wos and I undertook to make another attempt. One aim of this project is to produce a formal proof of each theorem ("Satz") in Szmielew, using as hypotheses (some of) the previous theorems and definitions, with the ultimate aim of formalizing the definitions and properties of multiplication, addition, and square root.

A second aim of the project is to see how much the techniques available for automated deduction have improved in the 20 years since Quaife. Would we now be able to solve the challenge problems that were left unsolved at that time?

And ultimately, a third aim of the project is to reach the propositions of Euclid, but based on Tarski's axioms.

\subsection{Szmielew in Otter}

Larry Wos and I experimented with going through Szmielew's development, making each theorem into an Otter file, giving Otter the previously proved theorems to use. We also used Prover9 sometimes, but we found it did not perform noticeably better (or worse) on these problems. Our aim was to obtain Otter proofs of each of Szmielew's theorems.

Some basic facts about Otter (and Prover9) will be helpful. Otter gives a weight to every formula (by default, the total number of symbols). You can artificially adjust the weights using a list called the "weight list". There is a parameter called max_weight; formulas with larger weights are discarded to keep the search space down. Thus you can control the search to some degree by assigning certain formulas low weights and finding a good value of max_weight that allows a proof to be found: just large enough that all needed formulas are kept, not so large that the prover drowns in irrelevant conclusions. These remarks apply to both Otter and Prover9; the difference between the two provers lies in the algorithm for choosing the next clauses to be considered for generating new clauses.

One technique we used is called "giving Otter the diagram." This means defining a name for each of the points that need to be constructed. For example,

\footnotetext{
${ }^{12}$ William Richter has also checked Szmielew up through Satz 3.1 in miz3 (see http://www.math.northwestern.edu/ richter/TarskiAxiomGeometry.ml). He has also checked some proofs from Hilbert's axioms; the code is in hol_light/RichterHilbertAxiomGeometry/ in the HOL-Light distribution.
} 
if the diagram involves extending segment $a b$ beyond $b$ by an amount $c d$, you would add the line $q=\operatorname{ext}(a, b, c, d)$. The point of doing so is that $q$, being an atom, gets weight 1 , so terms involving $q$ will be more likely to be used, and less likely to be discarded.

We also used "hints" and "resonators" [34]. By this we mean the following:

(i) We put some of the proof steps (from the book proof) in as preliminary goals. We get proofs of some of them.

(ii) We put the steps of those proofs into Otter's weight list, giving them a low weight, to ensure that they be chosen quickly to make new deductions. The bound max_weight is then set to the smallest value that will ensure their retention. This prevents the program from drowning in new and possibly irrelevant conclusions.

The technique of resonators can be used in other ways as well. For example, if you are trying to prove $C$ and you have proofs of some lemma $A$ and a proof of $C$ from the assumption $A$, but you cannot directly get a proof of $C$, then put the steps of the two proofs you do have in as resonators. Very likely you will find the desired proof of $C$. Wos has also used resonators very successfully to find shorter proofs, once a long proof is in hand.

\subsection{What happened}

Our first observation was that it is necessary to give Otter the diagram, in the sense described above. Once we started doing that, we went through Chapters 2 and 3 (of 2-16) rapidly and without difficulty.

We hit our first snag at Satz 4.2. An argument by cases according as $a=c$ or $a \neq c$ is used. Otter could do each case, but not the whole theorem! We tried Prover9. Prover 9 could prove Satz 4.2, but it took 67,000 seconds! (1 day = 86,400 sec.)

The inability to argue by cases is a well-known problem in resolution theoremproving. On perhaps ten (out of more than 100) subsequent theorems, we had to help Otter with arguments by cases. Sometimes we did that by putting in the case split explicitly, and giving the cases low weights. For example we would put in $b=c \mid b !=c$ and then give both literals a negative weight. With this trick, if the cases can be done in separate runs, we could sometimes get a proof in a single run. ${ }^{13}$ If not, then we used the proof steps of both cases as resonators.

Chapter 5 of [29] contains a difficult theorem from Gupta's thesis [12], the connectivity of betweenness (Satz 5.1). That theorem is

$$
a \neq b \wedge T(a, b, c) \wedge T(a, b, d) \rightarrow T(a, c, d) \vee T(a, d, c) .
$$

\footnotetext{
${ }^{13}$ Ross Overbeek suggested a general strategy: if you don't get a proof, look for the first unit ground clause deduced, and argue by cases (in two runs) on that clause. That strategy would have worked on Satz 4.2. Here is a project: implement this strategy using parallel programming.
} 
Neither Otter nor Prover9 could prove Gupta's theorem without help. We used resonators, starting with about thirty of Gupta's proof steps. This technique was successful. We found a proof of Satz 5.1.

After proving the connectivity of betweenness, we had no serious difficulties with the rest of Chapters 5 and 6; Otter required no help except a couple of case splits.

In 1990, Quaife made a pioneering effort (using Otter) to find proofs in Tarski's geometry. He used the version of Tarski's axioms [29], just as we do. Quaife made it a bit farther than where Wos and I hit our first snag in Szmielew. Most of Quaife's theorems are in Szmielew Chapters 2 and 3, or the first part of 4 , or are similar to such theorems, but use some defined notions such as "reflection," which occurs in Chapter 7 of [29]. His most difficult example was that the diagonals of a "rectangle" bisect each other. Here a "rectangle" is a quadrilateral with two opposite sides equal and the diagonals equal. This theorem is weaker than Lemma 7.21 in [29], which says that in a quadrilateral in which (both pairs of) opposite sides are congruent, the diagonals bisect each other.

Quaife left four challenge problems, which are theorems in that part of [29] that we formalized. Although he did not say so explicitly, it is clear that these should be solved from axioms A1-A9, i.e. without the parallel axiom or any continuity assumptions. We list them here:

- the connectivity of betweenness (Satz 5.1 in [29])

- every segment has a midpoint (Satz 8.22 in [29]).

- inner Pasch implies outer Pasch (Satz 9.6 in [29])

- Construct an isosceles triangle with a given base (an immediate corollary of Satz 8.21 and Satz 8.22, the existence of midpoints and perpendiculars).

The difficulty of proving the existence of a midpoint lies in the fact that no continuity axioms are allowed. (The usual construction using two circles is thus not applicable.) This requires developing the theory of right angles and perpendiculars, and takes most of Chapters 7 and 8 of [29]. The construction depends on two difficult theorems: the construction of a perpendicular to a line from a point not on the line, and the construction of a perpendicular to a line through a point on the line (Satz 8.18 and 8.20). These in turn depend on another theorem of Gupta, called the "Krippenlemma" (Lemma 7.22). The proof that inner Pasch implies outer Pasch is one of the highlights of [12].

\subsection{Our results}

We were eventually able to prove all four of Quaife's challenge problems, and indeed all the results from [29] up to and including Satz 9.6. The proofs we found, the Otter input files to produce them, and some discussion of our techniques, are available at [2]. Satz 7.22 (the Krippenlemma) and Satz 8.18 (construction of the perpendicular) were extremely difficult, and required many iterations of proofs of intermediate results, and incorporation of new resonators from those proofs. We could never have found these Otter proofs without the aid of Gupta's proofs, 
so in some sense this is "computer-assisted deduction", intermediate between "proof-checking" and "automated deduction."

Why were we able to do better in 2012 than Quaife could do in 1990? Was it that we used faster computers with larger memories? No, it was that we used techniques unknown to Quaife. We could not find these proofs with 2012 computers using only Quaife's techniques. Maybe we could have found the proofs we found with 1990 computers and 2012 techniques, but we're glad we didn't have to. Quaife knew how to tell Otter what point to construct (and we learned the technique from him), but he didn’t know about resonators.

\subsection{Euclid from Tarski}

As of summer 2012, neither by hand nor by machine had development from Tarski's axioms reached the first proposition of Euclid, more than half a century after Tarski formulated his axioms, although an approach from a far less parsimonious axiom set has allowed the mechanization of some of Euclid [1]. Quaife did not get as far as proving any theorem about circles. Neither did Szmielew or Gupta. All these authors wanted to postpone the use of even line-circle or circle-circle continuity as long as possible, while Euclid uses it (implicitly) from the outset. We felt that it was high time to prove at least the first proposition of Euclid from Tarski's axioms.

Euclid's Book I, Prop. 1. constructs an equilateral triangle, as shown in Fig. 7. The open circle indicates the constructed point. Euclid's proof does not meet the modern standards of rigor, according to which one would need some sort of continuity axiom and perhaps some sort of dimension axiom to prove Prop. 1, since if the circles were in different planes, they would not meet. It turns out that the dimension axioms are not needed, because "circle-circle continuity" is sphere-sphere continuity in $\mathbb{R}^{3}$.

Fig. 7. Euclid Book I, Proposition 1

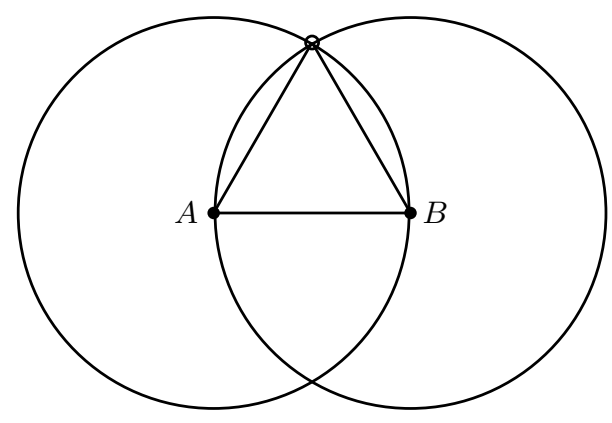


The result: Otter proves Euclid I.1 from circle-circle continuity in less than two seconds, with a good choice of inference rules. When we first did it, it took eleven minutes, which is about how long it will take you by hand.

Euclid's Prop I.2 says that given three points $A, B$, and $C$, a point $D$ can be constructed such that $A D=B C$. That is immediate from Tarski's segment extension axiom (A4). Euclid only postulated you can extend a segment somehow, so in a sense, Tarski's (A4) is unnecessarily strong.

Euclid's Prop I.3 mentions the concepts "the greater" and "the lesser" between segments. In Tarski's primitives, we would define $a b \leq c d$ to mean that for some $x$ we have $a b \equiv c x$ and $T(c, x, d)$. Then Prop. I.3 has no content; in other words Prop. I.3 amounts to a definition of "the greater" and "the lesser", which in Euclid are "common notions."

Prop. I.4 is the SAS congruence criterion. That requires defining angle congruence; it is Satz 11.4 in Szmielew! There is a big jump from the first three propositions to Prop. I.4. The reason is that angle congruence and indeed comparison of angles ( $\leq$ for angles) are primitive in Euclid, but defined in Tarski's system. The resulting complications have little to do with automated deduction. They are the consequence of choosing a very parsimonious formal language. Therefore, we should complete Szmielew Chapter 11 first, or take some axioms about comparison and congruence of angles, in order to formalize Euclid directly. (That is the approach taken in [1].) We plan to return to Euclid after finishing the formalization of Szmielew up to Chapter 11. For example, Euclid's Prop. I.8 is the SSS angle criterion, Satz 11.51 in Szmielew.

\section{Proof by computation, in theory and practice}

In this section, we discuss the top and right sides of the diagram. The plan, in theory, is to verify the truth of (which in a loose sense is to prove), some geometric formula $A$. We start by expressing it as a system of algebraic equations (or inequalities) using analytic geometry (or by some other method), introducing new variables for the coordinates of the points to be constructed (or some other quantities depending on those points). Then we calculate to see if these equations can be satisfied. If the calculation succeeds, then $A$ is verified. But we still do not have a first-order proof of $A$.

To put this method into practice, we need to answer two questions: Exactly how will we convert from geometry to algebra, and exactly how will we make the required computations? Among the ways to convert geometry to algebra, we mention the ordinary introduction of coordinates, and Wu's method [35], and Chou's area method [8]. Among the ways to compute, we mention Gröbner bases and the Collins CAD algorithm $[7,6]$. While theoretically, any geometry problem can be solved by $\mathrm{CAD}$, since it is a decision procedure for real-closed fields, in practice, it breaks down on problems with five or six (number) variables, so a geometry problem with four points is likely to be intractable, and geometry problems with fewer than four points are rare. On the other hand, Wu's method and Chou's area method have been used to prove hundreds of beautiful theorems, 
some of them completely new. In that sense, they far outperformed resolution theorem proving.

In spite of the dramatic successes of these methods, we point out two shortcomings. First, both these methods work only on theorems that translate to algebra using equations, with no inequalities. Thus the "simple" betweenness theorems of Szmielew Chapter 3 are out-of-scope.

Second, you cannot ask for a proof from ruler-and-compass axioms (or indeed from any geometric axioms at all). You can only ask if the theorem is true in $\mathbb{R}^{2}$. Thus there is no problem trisecting an angle; this is not about ruler-andcompass geometry. A proposition like Euclid I.1 is just trivial: all the subtleties and beauties of the first-order proof are not captured by these methods. It just computes algebraically that there is a point on both circles.

In short, when using these methods, we are not doing geometry. We are doing algebra. It is these shortcomings that we propose might be rectified, if we could make the diagram commute in practice. Then we could go along the bottom of the diagram, benefiting from computation on the right, and still end up with geometrical proofs on the left.

In theory, we should be able to get geometric proofs by going across the bottom of the diagram from right to left. That is, to convert the algebra performed by Chou's method into first-order proofs of algebraic theorems, from some algebraic axioms, and then back-translate to geometry, using the geometric definitions of addition and multiplication. In theory this can certainly be done. In practice, the authors of [8] were aware of this possibility, and discuss it on pp. 59-60, but they say, "The geometric proofs produced in this way are expected to be very long and cumbersome, and as far as we know no single theorem has been proved in this way." Nevertheless, those proofs, if we could find them, would be proofs and not just computations.

\section{From computation to proof: going around the dragons}

Here is the plan to find a first-order proof of a given geometric theorem by going across the top of the diagram, down the right, and back, all within VG.

- Start with a geometric theorem $\phi$ to be proved.

- Do the analytic geometry to compute $\phi^{\star}$. (By Chou or Descartes)

- Find (e.g. by Chou's program or by hand) an informal proof that $\phi^{\star}$ is true, by calculation.

- Get a formal proof in VG of $\phi^{\star}$, i.e., verify the calculation.

- Use (an implementation of) Theorem 1 to get a proof of $\phi$ in VG.

- Eliminate the non-geometrical axioms to get a proof of $\phi$. This can be done, at least in theory, by (an implementation of) Theorem 3.

The main point to be made about this plan is that the difficulty is essentially a "boot-strapping" issue. To get started, we need (machine) formalization of the geometric definitions of addition, multiplication, and square root. These proofs 
need to be produced just once, and then we can use them to find proofs of many different geometrical theorems. The central importance of the theorems justifying these definitions has long been recognized, as these theorems are in some sense the culminating results of both [14] and [29]. Indeed, the key to proving the properties of multiplication (no matter whether one uses the definition of Descartes or that of Hilbert) is the theorem of Pappus (or Pascal as Hilbert called it). Even the commutativity of addition is not completely trivial. Chapter 15 of Szmielew [29] has the details.

Narboux [20] tried formalizing [29] in Coq, but he didn't get to Chapter 15. Wos and I tried it with Otter, as reported here, but we didn't get to Chapter 15 (yet) either. It turned out that using Otter was not as efficient in finding formal proofs as we had hoped, many human hours were also required. Our hope that every theorem in [29] would be a single, easy run with Otter turned out not to be justified; while that was true of the simpler theorems, every theorem complex enough to require a diagram required several runs, case distinctions made by hand, points defined, and the use of resonators made from lemmas or partial results. As mentioned above, Coq does not produce first-order proofs, and it is probably not easy to extract them from Coq proofs.

\section{A test case: the centroid theorem (medians all meet)}

We propose a test case for the back-translation method, once someone manages to formalize the definitions of addition, multiplication, and square root. Namely, the theorem that all the medians of a triangle meet in a single point; this is known as the "centroid theorem." Perhaps it is possible to prove this theorem formally from Tarski's axioms using theorems of [29], but that would not count as a solution of this test case.

When Chou's area method is applied to this example, the computations are quite simple (see [8], p. 12); even Cartesian analytic geometry is not very complicated. What is required are the following steps:

- Formalize the geometry-to-algebra reasoning in VG.

- Formalize the algebraic computation in VG.

- Carry out the back-translation and get a formal proof in EG.

That proof would no doubt be long and not very perspicuous. One of the reasons we chose Otter, is that at this point, we could shorten that long proof, using Wos's proof-shortening techniques. Perhaps the complications would melt away, leaving a short proof. Or perhaps the proof would remain impenetrable; we cannot know without performing the experiment. We note, however, that even if the long proof is obtained by another tool, it could still be translated into Otter's language for attempts at proof-shortening.

\section{Four challenge problems}

Having solved some of Quaife's challenge problems, we offer four more. Of course, it goes (almost) without saying that it is a challenge to finish the formalization 
of [29]; we mean four in addition to that. Our first three challenge problems involve line-circle continuity $(\mathbf{L C})$ and circle-circle continuity $(\mathbf{C C})$. These three problems are

$$
\begin{array}{ll}
\mathbf{C C} \rightarrow \mathbf{L C} & \text { using A1-A9 only } \\
\mathbf{L C} \rightarrow \mathbf{C C} & \text { using A1-A10 } \\
\mathbf{L C} \rightarrow \mathbf{C C} & \text { using A1-A9 only }
\end{array}
$$

A first-order proof that $\mathbf{C C}$ implies LC using A1-A9 is sketched on pages 200202 of [11]. The other direction, $\mathbf{L C} \rightarrow \mathbf{C C}$, is more difficult. If we allow the use of the parallel axiom A10, then it is relatively easy to prove that implication model-theoretically. What has to be shown is that if $\mathbb{F}$ is a Pythagorean field, and $\mathbb{F}^{2}$ satisfies either one of the line-circle or circle-circle continuity, then $\mathbb{F}$ is a Euclidean field. This is done by ordinary analytic geometry; see [13], p. 144, with missing details supplied as in [3]. But that still doesn't give us a firstorder proof. With the aid of the parallel postulate (A10), the proof by analytic geometry could, in theory, be used with back-translation to get a first-order proof. In practice, we do not expect to be able to convert this model-theoretic proof to first-order in the near future, so it is a challenge to find a first-order proof by some other means.

In [30], Strommer showed that $\mathbf{L C} \rightarrow \mathbf{C C}$ can be proved without the parallel axiom. A model-theoretic proof, based on the Pejas classification of Hilbert planes [23], is also known; see the discussions in [11], p. 202 and [13], p. 110. Strommer's proof has the advantage of being first-order (although it is couched in terms of Hilbert's axiom system, not Tarski's). Strommer's proof can probably be made computer-checkable by proceeding through his paper one theorem at a time, but it is a challenge to do so.

The fourth challenge problem is as follows: Prove from A1-A10 that it is possible to construct an equilateral triangle on a given base. That is, prove Euclid I.1 without using circles. Hilbert raised the problem of making such a construction, and gave a solution, in his 1898 "vacation course" [15], page 169. Hilbert's solution is quite simple; it is based only on constructing perpendiculars and bisecting segments. One successively constructs right triangles with hypotenuses of length $\sqrt{2}, \sqrt{3}, \sqrt{3} / 2$, as shown in Fig. 8. Since Chapter 8 of Szmielew has the required constructions of perpendiculars and midpoints, we might be in a position to try to get an Otter proof corresponding to Hilbert's construction. But there is a piece of analytic geometry at the end, involving the Pythagorean theorem, which requires the parallel postulate A10. To get a proof from A1-A10, one will certainly have to use the parallel axiom, because the theorem (as Hilbert knew) is not true in all Hilbert planes, i.e. does not follow from A1-A9. See for example [13], Exercise 39.31, p. 373. We tried this problem by giving Otter the diagram for Hilbert's construction, but so far to no avail. We could only apply a 1990 technique, because we have no idea what resonators to use. If the program suggested in this paper could be carried through, we could back-translate Hilbert's proof from analytic geometry to A1-A10. 
Fig. 8. Constructing an equilateral triangle without using circles

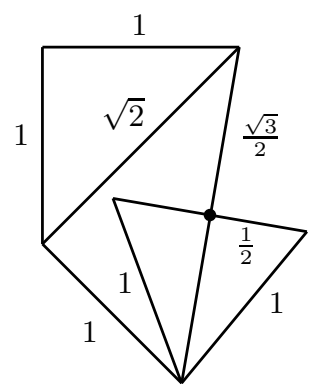

\section{References}

1. Avigad, J., Dean, E., Mumma, J.: A formal system for Euclid's Elements. Review of Symbolic Logic 2, 700-768 (2009)

2. Beeson, M.: www.michaelbeeson.com/research/FormalTarski/index.php

3. Beeson, M.: Foundations of Constructive Geometry. Available on the author's website, www.michaelbeeson.com/research/papers/pubs.html (2012)

4. Beeson, M.: Logic of ruler and compass constructions. In: Cooper, S.B., Dawar, A., Loewe, B. (eds.) Computability in Europe 2012. Springer (2012)

5. Borsuk, K., Szmielew, W.: Foundations of Geometry: Euclidean and BolyaiLobachevskian Geometry, Projective Geometry. North-Holland, Amsterdam (1960), translated from Polish by Erwin Marquit

6. Brown, C.W.: QEPCAD B, a program for computing with semi-algebraic sets using cads. SIGSAM Bulletin 37, 97-108 (2003)

7. Caviness, B.F., Johnson, J.R. (eds.): Quantifier Elimination and Cylindrical Algebraic Decomposition. Springer, Wien/New York (1998)

8. Chou, S.C., Gao, X.S., Zhang, J.Z.: Machine Proofs in Geometry: Automated Production of Readable Proofs for Geometry Theorems. World Scientific (1994)

9. Feferman, S. (ed.): The Collected Works of Julia Robinson. American Mathematical Society (1996)

10. Fischer, M.J., Rabin, M.O.: Super-exponential complexity of Presburger arithmetic. SIAM-AMS Proceedings VII, 27-41, reprinted in [7], pp. 27-41 (1974)

11. Greenberg, M.J.: Old and new results in the foundations of elementary plane euclidean and non-euclidean geometries. American Mathematical Monthly 117, 198219 (March 2010)

12. Gupta, H.N.: Contributions to the Axiomatic Foundations of Geometry. Ph.D. thesis, University of California, Berkeley (1965)

13. Hartshorne, R.: Geometry: Euclid and Beyond. Springer (2000)

14. Hilbert, D.: Foundations of Geometry (Grundlagen der Geometrie). Open Court, La Salle, Illinois (1960), second English edition, translated from the tenth German edition by Leo Unger. Original publication date, 1899.

15. Hilbert, D.: David Hilbert's lectures on the foundations of geometry 1891-1902. Springer-Verlag, Berlin Heidelberg New York (2004), edited by Michael Hallett and Ulrich Majer

16. Kempe, A.B.: On the relation between the logical theory of classes and the geometrical theory of points. Proceedings of the London Mathematical Society 21, 147-182 (1890) 
17. Marchisotto, E.A., Smith, J.T.: The Legacy of Mario Pieri in Geometry and Arithmetic. Birkhauser, Boston, Basel, Berlin (2007)

18. McCharen, J., Overbeek, R., Wos, L.: Problems and experiments for and with automated theorem-proving programs. IEEE Transactions on Computers C-25(8), 773-782 (1976)

19. Mollerup, J.: Die Beweise der ebenen Geometrie ohne Benutzung der Gleichheit und Unbleichheit der Winkel. Mathematische Annalen 58, 479-496 (1904)

20. Narboux, J.: Mechanical theorem proving in Tarski's geometry. In: Botana, F., Recio, T. (eds.) Automated Deduction in Geometry: 6th International Workshop, ADG 2006, Pontevedra, Spain, August 31-September 2, 2006, Revised Papers. pp. 239-156. Lecture Notes in Artificial Intelligence, Springer (2008)

21. Pasch, M.: Vorlesung über Neuere Geometrie. Teubner, Leipzig (1882)

22. Pasch, M., Dehn, M.: Vorlesung über Neuere Geometrie. B. G. Teubner, Leipzig (1926), the first edition (1882), which is the one digitized by Google Scholar, does not contain the appendix by Dehn.

23. Pejas, W.: Die Modelle des Hilbertschen Axiomensystems der absoluten Geometrie. Mathematische Annalen 143, 212-235 (1961)

24. Pieri, M.: La geometry elementare istituita sulle nozioni di "punto" e "sfera" (elementary geometry based on the notions of point and sphere). Memorie di matematica e di fisica della Società Italiana delle Scienze 15, 345-450 (1908), english translation in [17], pp. 160-288

25. Quaife, A.: Automated development of Tarski's geometry. Journal of Automated Reasoning 5, 97-118 (1989)

26. Quaife, A.: Automated Development of Fundamental Mathematical Theories. Springer, Berlin Heidelberg New York (1992)

27. Renegar, J.: Recent progress on the complexity of the decision problem for the reals. DIMACS Series 6, 287-308, reprinted in [7], pp. 220-241 (1991)

28. Robinson, J.: Definability and decision problems in arithmetic. Journal of Symbolic Logic 14, 98-114, reprinted in [9], pp.7-24 (1949)

29. Schwabhäuser, W., Szmielew, W., Tarski, A.: Metamathematische Methoden in der Geometrie: Teil I: Ein axiomatischer Aufbau der euklidischen Geometrie. Teil II: Metamathematische Betrachtungen (Hochschultext). Springer-Verlag (1983), reprinted 2012 by Ishi Press, with a new foreword by Michael Beeson.

30. Strommer, J.: über die Kreisaxiome. Periodica Mathematica Hungarica 4, 3-16 (1973)

31. Tarski, A.: What is elementary geometry? In: Henkin, L., Suppes, P., Tarksi, A. (eds.) The axiomatic method, with special reference to geometry and physics. Proceedings of an International Symposium held at the Univ. of Calif., Berkeley, Dec. 26, 1957-Jan. 4, 1958. pp. 16-29. Studies in Logic and the Foundations of Mathematics, North-Holland, Amsterdam (1959), available as a 2007 reprint, Brouwer Press, ISBN 1-443-72812-8

32. Tarski, A., Givant, S.: Tarski's system of geometry. The Bulletin of Symbolic Logic 5(2), 175-214 (June 1999)

33. Veblen, O.: A system of axioms for geometry. Transactions of the American Mathematical Society 5, 343-384 (1904)

34. Wos, L.: Automated reasoning and the discovery of missing and elegant proofs. Rinton Press, Paramus, New Jersey (2003)

35. Wu, W.T.: Mechanical Theorem Proving in Geometries: Basic Principles. SpringerVerlag, Wien/ New York (1994)

36. Ziegler, M.: Einige unentscheidbare körpertheorien. Enseignement Math. 2(28), 269-280 (1982) 